Bull. Egypt. Soc. Physiol. Sci. Vol. (41) Issue (2), 140- 154

\author{
Bull. of Egyp. Soc. Physiol. Sci. \\ (Official Journal of Egyptian Society for Physiological Sciences) \\ (pISSN: 1110-0842; eISSN: 2356-9514)
}

\title{
Effect of Intra-Articular Injection of Chondrocytes Differentiated from Mesenchymal Stem Cells in Monosodium iodoacetate Induced Osteoarthritis in Male Rats
}

\author{
Maha Mohammed Elbatsh ${ }^{1 *}$ Rawhia Hassan Eledel ${ }^{2}$ Rasha Ibrahim Noreldin ${ }^{2}$ \\ Asmaa Shams El Dein Mohamed ${ }^{3}$ and Thoria Ahmed Omar ${ }^{2}$ \\ ${ }^{1}$ Clinical Pharmacology department, Faculty of Medicine, Menoufia University, Shebin ElKom, Egypt \\ ${ }^{2}$ Clinical pathology department, Faculty of Medicine, Menoufia University, Shebin ElKom, Egypt \\ ${ }^{3}$ Pathology department, Faculty of medicine, Menoufia University, Shebin ElKom, Egypt
}

Submit Date: May 7, 2020

Accept Date: July 27, 2020

Available online: Jan 10, 2021

\section{Keywords}

- Wharton jelly,

- Mesenchymal stem

cells

- Chondrocytes

- Osteoarthritis

\begin{abstract}
Background: Osteoarthritis $[\mathrm{OA}]$ is a chronic, prevalent, debilitating joint disease characterized by progressive cartilage degradation, subchondral bone remodeling, bone marrow lesions, meniscal damage, and synovitis. Joint diseases are very difficult to be treated with traditional line therapy. Hence the importance of developing new therapeutic methods such as stem cell therapy. Mesenchymal Stem Cells [MSCs] isolated from multiple tissues as bone marrow, adipose tissue, dental pulp, placenta and umbilical cord. They possess ability of self-renewal and multilineage differentiation which is a hoping tool for treatment of multiple diseases. The aim of this study is to assess the ability of stem cells to differentiate into chondrocytes and the ability of those chondrocytes to induce cartilage repair and suppresses cartilage degeneration in Monosodium iodoacetate [MIA] induced osteoarthritis in rats' model. Materials: In this experimental study Wharton jelly separated from umbilical cord was used to isolate MSCs then differentiated to chondrocytes and injected into MIA induced osteoarthritis in rat's model. The rats divided into three groups; control group, MIA-treated group and MIA- Chondrocytes treated group. Results: MIA- Chondrocytes treated group appeared healthy with no signs of inflammation. On microscopic examination: the articular cartilage of injected limbs showed observable restoration of surface continuity, chondrocytes density, boundaries between layers and matrix basophilia. Conclusions: Intra-articular injection of chondrocytes improves, inhibits the progression of degeneration of the affected cartilage and induces cartilage repair.
\end{abstract}

Corresponding author: Dr Maha Mohamed ElBatsh: Assistant Professor of Clinical Pharmacology , Clinical Pharmacology Department Faculty of Medicine, Menoufia University, Shebin ElKom, Egypt Zip code 32511, maha.ali@,med.menofia.edu.eg 


\section{INTRODUCTION}

Osteoarthritis (OA) is a rheumatic joint disorder. It considers the most common form of arthritis. It is chronic, prevalent, debilitating joint disease suspected in people over the age of 45 or older. It is characterized by erosion of articular cartilage and reactive new bone formation at the articular boundaries, initiating pain and rigidity in the affected joints [1]. OA can affect all synovial joints but the main joints affected are: hands, fingertips, knees, hips and spine of the neck or lower back [2]. The most common symptoms of OA include pain, tenderness, stiffness, inflammation, and locomotors restriction. When OA progressed, it associated with more severe pain, swelling in the joint and surrounding area may also occur and this is frequently associated with depression and sleeping disorder [3]. Current strategy for OA treatment is centered upon symptom management and can't reverse the damage of OA. New therapeutic approaches used for $\mathrm{OA}$ are targeted for restoration of articular cartilage, intra-articular ligaments, and menisci as it's has no capability to regenerate [4]. Stem cells are the sources of tissue repair, regeneration and a promising source for novel therapies. The present stem cell therapies use mesenchymal stem cells (MSCs), as it carries the remarkable capability of differentiation into diverse cell types while retaining their capability of self-replication and maintaining the characteristics of their parent cells. This could be effective in repairing the damaged joints, cartilage and remodeling subchondral bone in OA [5]. Stem cells (SC) are biological cells establish in nearly all multicellular organisms. Stem cells are cells with the potential to develop into many different types of cells in the body. They serve as a repair system for the body [6]. Mesenchymal stem cells (MSCs) are multipotent stromal cells that can differentiate into a variety of cell types, including bone cells, cartilage cells, muscle cells, fat cells, liver cells, and epithelial cells [7]. It has immunomodulatory, antiinflammatory and trophic effects [8]. Bone marrow (BM) and adipose tissue (AT) are still the most frequently sources for the separation of MSCs. All postnatal tissues such as placenta, umbilical cord blood and tissue have also shown promising sources for isolation and proliferation into MSCs [9].

Wharton's jelly of the umbilical cord considers a source of MSCs which is able to differentiate into osteogenic, adipogenic, neural, chondrogenic and cardiogenic cells [10]. Wharton's jelly- MSCs (WJ-MSCs) have many advantages, including non-invasive, nontumorigenicity, easy harvest ability, increased proliferative capacity and accessibility in large numbers [11].

WJ-MSCs express both an immunoprivileged and immunomodulatory phenotype and their Major histocompatibility complex (MHC) class I expression levels can be manipulated, while Major histocompatibility complex class II is negative, making them a potential cell source for MSC- based therapies. In addition, these cells represent a non-controversial source of primitive mesenchymal progenitor cells that can be harvested after birth, cryogenically stored, thawed, and expanded for therapeutic uses [13]. The potential use of stem cell-based therapies for the repair and regeneration of several tissues and organs offers a paradigm shift that may provide 
alternative therapeutic solutions for many of bone diseases [14].

MSCs are heterogeneous group of cells with different growth potential, distinctive morphologic and functional characteristics, all have chondrogenic potential and potent immunosuppressive properties representing a potent tool in the treatment of OA [15].

MSCs contribute to cartilage repair through chondrogenic differentiation, the production of paracrine factors and extracellular vesicles or immunomodulatory effect [16]. Therefore, we hypothesize that chondrocytes differentiated from WJ-MSCs could demonstrate regenerative cellular effect in MIA model of osteoarthritis. This idea was investigated before but with use WJ-MSCs directly without differentiation, so we not found enough data about use differentiated chondrocytes in treatment osteoarthritis. Up to our knowledge it is the first time to inject chondrocytes in ankle joint of rat model of osteoarthritis.

This study aimed to isolate MSCs from human Wharton jelly of umbilical cord and evaluate the potential of their differentiation into chondrocytes. Monosodium iodoacetate (MIA) was used to induce OA-like lesion in joints of rats. These lesions manifest characteristic OA features such as cartilage degradation, subchondral bone sclerosis, and long- lasting pain. Here, we evaluated the effects of weekly intra-articular injections of MSC in the rat model and investigated the restoration of matrix homeostasis in ankle repair and regeneration.

\section{Materials and methods}

This experimental study was conducted at Clinical Pathology department in collaboration with Obstetrics \& Gynecology, Pharmacology and
Pathology departments at Menoufia University during the period from May 2017 to April 2019 after obtaining Menoufia ethical committee approval.

Umbilical cord samples from normal pregnant females coming for delivery were involved. Females with known history of hepatitis, infectious diseases, diabetes mellitus, severe hypertension, abortions or bad obstetric history were excluded from collection. Cord samples were aseptically collected from patients after obtaining their consent.

\subsection{Wharton's jelly (WJ- MSCs):}

MSCs were isolated from WJ by an explant method [17]. Cords were washed in PBS then cut into small pieces $(0.5-1 \mathrm{~cm})$. Blood vessels were detached to avoid endothelial cell contamination. The WJ pieces were sited immediately into culture flasks for culture expansion in low glucose DMEM containing 10\% CBS, Penicillin $(500 \mu \mathrm{g})$ and Fungizone $(0.25 \mu \mathrm{g} / \mathrm{mL})$. After 14 days; when cell colonies appeared, and reached $70 \%$ confluence, cells were suspended in $0.25 \%$ trypsin-EDTA $(0.25 \% \operatorname{tryp} \sin / 0.1 \%$ EDTA $)$ and reseeded at a concentration of $2 \times 10^{3} \mathrm{cells} / \mathrm{cm}^{2}$. These reagents were purchased from Lonza (8830 Biggs Ford Rood, Walkersville, MD21793, USA)

\subsubsection{Characterization of WJ-MSCs Surface Marker}

Immunophenotyping of WJ-MSCs was performed by Flow Cytometric analyzer. Trypsinized cells were washed and suspended in PBS at a density of $1 \times 10^{7}$ cells $/ \mathrm{mL}(100 \mu \mathrm{L} /$ tube $)$. The cells were then incubated with antibodies ( anti-CD73/FITC, anti-CD44/PE, anti-CD45/FITC, anti- and $\mathrm{CD} 34 / \mathrm{PE}$ ) on ice for $15 \mathrm{~min}$ in the dark. 
MSCs were splashed 2 times with $2 \mathrm{~mL}$ of PBS with $1 \%$ BSA then centrifuged at $3200 \mathrm{rpm}$ for 5 minutes. The nonspecific mouse $\mathrm{IgG}$ was substituted for the primary antibodies as isotype control. These cells were analyzed with a BD FACS Calibur flow cytometer (BD Biosciences). CellQuest for Mac v3.0 (BD Biosciences) was used to analyze the phenotypes of the cells. All antibodies were purchased from BD Biosciences (BD Biosciences, San Jose, CA, USA), included the appropriate isotype controls.

\subsubsection{Induction of Osteogenic, Adipogenic, and Chondrogenic Differentiation [18]}

All chemicals and reagents were purchased from Sigma-Aldrich Corporation (St. Louis, MO, USA), unless otherwise indicated.

To determine the differentiation potential of hWJMSCs, MSCs incubated with LG- DMEM proliferation medium for 14 days and when 60$70 \%$ as described before and immediately subjected to adipogenic, osteogenic and Chondrogenic growth factors, at the end of the expansion process without detaching the cells from the surface

After $2^{\text {nd }}$ passage and when hWJ-MSCs reach 60$70 \%$ confluence, the differentiation potential of MSCs were determined.

Adipogenic differentiation: hWJ-MSCs were cultured in basal medium with CBS [5\%], complemented with insulin $\left[\begin{array}{ll}10 & \mu \mathrm{g} / \mathrm{m}\end{array}\right]$, indomethacin $[100 \mu \mathrm{M}]$, dexamethasone $[1 \mu \mathrm{M}]$, isobutylmethylxanthine (IBMX) [0.5 mM]. IBMX was eliminated from medium after 7 days. The medium was changed every 2 days for 21 days.

Osteogenic differentiation: MSCs were fed with low glucose-DMEM supplied with reduced CBS to $5 \%, 1 \%$ penicillin/streptomycin/ fungizone. Media enhanced with dexamethasone $[100 \mathrm{nM}]$, Lascorbate2-phosphate $[0.2 \mathrm{mM}]$, and $\beta$ glycerophosphate $[10 \mathrm{mM}]$. The medium was changed every 2 days for 21 days.

\subsubsection{Chondrogenic differentiation: hWJ-MSCs}

were relocated into $15 \mathrm{~mL}$ Falcone tubes and spun down. MSCs cultured in a chondrogenic medium composed of low glucose-DMEM supplied with $2 \% \quad \mathrm{CBS} \quad, 1 \% \quad$ Insulin-Transferrin-SeleniumEthanolamine (ITS-X, Invitrogen), $50 \mu \mathrm{g} / \mathrm{mL}$ ascorbate-2-posphate, $40 \mu \mathrm{g} / \mathrm{mL}$ L-proline, 100 $\mu \mathrm{g} / \mathrm{mL}$ sodium pyruvate, $100 \mathrm{nM}$ dexamethasone, and $10 \mathrm{ng} / \mathrm{mL}$ of TGF- $\beta 3$ (Prospec, East Brunswick, NJ, USA). $1 \mathrm{~mm}^{3}$ pellet was formed after overnight incubation of MSCs with chondrogenic medium. Differentiation medium was successively exchanged each 2-3 days for 3 weeks.

After $1^{\text {st }}$ passage and when cells reach $70-80-\%$ confluence a panel of antibodies containing; antimFABP4, anti-hOsteocalcin and anti-hAggrecan, was tested by immunofluorescence to define the mature phenotypes of adipocytes, osteocytes and chondrocytes respectively (R\&D Systems, USA).

For adipogenic, osteogenic and chondrogenic lineages, immunocytochemical (ICC) staining was done on days 18, 19 and 21 respectively. The chondrocytes pellet was splashed with PBS and fixed with zinc formalin solution then crysectioned (Histoserv, Inc., Germantown, MD) to slices about $5 \mathrm{~mm}$ thickness and mounted on microscope slide for examination. The tissue slides were fixed in $4 \%$ formaldehyde, permeabilized with $0.3 \%$ Triton-100X, and blocked with $1 \%$ BSA and 10\% normal donkey serum (NDS). Differentiated cells were incubated with primary antibodies for 3 hours at room temperature. For 
adipogenic and chondrogenic lineages, secondary NL557- conjugate donkey anti-goat IgG antibodies (R \&D System catalog \#NL001) at 1: 200 dilutions were incubated for 60 minutes, and washed before imaging. For the osteogenic lineage, NL557conjugate donkey anti-mouse IgG antibodies ( R \&D System catalog \#NL007) at 1: 200 dilutions were applied to the tissue slide under similar incubation conditions. DAPI used as counterstain for 5 minutes, the tissue slides were washed and mounted on an Inverted Fluorescence Microscope (Olympus IX70, Olympus, Tokyo, Japan) for imaging.

\subsection{Animals and experimental design:}

The present experiment was carried out with 24 adult male Sprague Dawley rats weighing 150-200 grams obtained from animal house of Tanta Faculty of Pharmacy. They were housed in standard cages (4 rats/cage) under room temperature $\left(22-24{ }^{\circ} \mathrm{C}\right)$ with humidity $(30-40 \%)$. The rats were exposed to 12 hours light and 12 hours dark cycle. They had free access to water and diet. The rats were acclimatized to these laboratory conditions seven days prior to the experiment. All procedures were carried out in accordance with the Public Health Service Policy on Use of Laboratory Animals published by the National Institutes of Health and were approved by the Ethical Committee of the College of Medicine, Menoufia University, Egypt.

Chondrocyte cells were harvested to be used in the animal experiment. The rats were randomly divided into 3 equal groups ( $\mathrm{n}=8$ each):

- Group 1 (control group): received vehicle treatment.
- Group 2 (MIA-treated group): received intraarticular injection of MIA $(0.3 \mathrm{mg} / 50 \mathrm{ul})$ in their left ankle.

- Group 3 (MIA- Chondrocytes treated group): Rats with OA treated with intraarticular injection chondrocytes cells at density of 500,000 cells per $0.5 \mathrm{~cm}$ sterile saline.

\subsubsection{Induction and treatment of $\mathrm{OA}$ :}

Rats were anesthetized with diethyl ether. Unilateral OA was induced in their left ankle by a single intra-articular injection of MIA (SigmaAldrich Corporation; St. Louis, MO, USA) at the dose of $0.3 \mathrm{mg}$ dissolved in $50 \mu \mathrm{l}$ of sterile physiologic saline solution through a $27 \mathrm{G}$ needle. We considered the rise of joint pressure due to intra-articular administration, so the same volume of saline was injected into the right ankle joint using the same method [19]. The control group received the same volume of saline intraarticular.

On day 21 rats with $\mathrm{OA}$ from group 3 were injected by chondrocytes cells at density of 500,000 cells per $0.5 \mathrm{~cm}$ sterile saline. Treatments were given 21 days after OA induction to permit the initial inflammatory phase after MIA injection to cease and structural damage to occur. Rats from group 1 and 2 received the same volume of sterile saline intraarticular. During the experiment; rats were observed for any gait changes. The body weight, ankle diameters and spontaneous locomotor activity were measured weekly. We examined the differences in the transverse and anteroposterior diameters of the affected and unaffected ankles using digital caliper with the ankle at $90^{\circ}$ dorsiflexion (neutral position). We measured each diameter three times at all-time points. Open field test has been used to evaluate the locomotor activity of rodents every week. The 
used open field apparatus was in the form of a square box with high walls $(100 \mathrm{~cm} \times 100 \mathrm{~cm} \times 50 \mathrm{~cm})$. All the inside walls had dark color and the floor of the area was divided by white lines into 25 equal squares [20]. In the day of experiment, each rat was placed in the center of the arena and left for 5 minutes to explore the arena freely. Rats' movements were recorded via video-camera located in the same room to be analyzed by two observers. Number of crossed squares were counted manually to record the horizontal locomotion. After each test the arena was cleaned then sprayed with $90 \%$ ethanol solution and wiped down with a clean paper towel. Rats were euthanized by decapitation 3 weeks after treatment, ankle joints were dissected for histological analysis. Histological changes were used to confirm the development of osteoarthritis as the intraarticular injection of MIA which is highly toxic causing mainly chondrocytes cell death and leading to cartilage degeneration.

\subsection{Histological evaluation:}

Three weeks after chondrocytes injection, all rats were sacrificed. The extracted joints were fixed in $20 \%$ formalin for 5-6 days. Each section was demineralized with acetone solution and ethylene diamine tetra acetic acid (EDTA) solution. It was fixed again with $20 \%$ formalin, decalcified with EDTA solution, and embedded in paraffin wax. The sections were stained with Hematoxylin and Eosin stains [21].

\subsection{Statistical analysis}

Results were statistically analyzed by SPSS version 22[SPSS Inc., Chicago, IL, USA]. Analytic statistics Paired- samples $T$ Test: is a test of significance used for comparison between two related groups normally distributed having quantitative variables. One- way ANOVA followed by Tukey's Multiple comparison test were used for analysis of changes in body weight. Two-way ANOVA and Bonferroni tests were used for analysis of ankle diameters and locomotor activity. Level of significance was set as P-value $\leq$ 0.05 .

\section{Results}

\section{Isolation and characterization of hWJ-MSCs}

WJ-MSCs were successively isolated from umbilical cord. MSCs were adherent to plastic flasks, spindle shape, and fibroblast-like cells. Cells growths were detected after one-week culture. After 14 days cells reached 70- 80\% confluence. For defining MSCs; three standard properties should be detected. First, MSCs should stick to the bottom of a plastic flask. When cultured up to14 days; the MSCs slowly exhibit a fibroblast-like, spindle-shaped morphology and collected together in a circinate appearance (Fig, 1), as detected by inverted phase contrast microscopy. Then, cultured MSCs were CD44, CD73, positive but negative for CD34 and CD45 (Fig, 2). Multilinage differentiation is a main property of hWJ-MSCs. To approve the in vitro multi-potency of hWJ-MSCs; MSCs induced to differentiate into tri-lineage: adipogenic, osteogenic and chondrogenic cells. After 3 weeks of culture in adipogenesis induction media, Cells induced developed lipid droplets that accumulated in the cells seen with inverted microscope. In osteogenic differentiation media for 21 days, the cells were acquired stellate- shape with cellular processes. The MSCs induced with the chondrogenic medium began to lose their characteristic fibroblastic shape and attained globular shaped chondrocyte-like-cells 1 week 
after adding chondrogenic differentiating media.

The globular cells continued to increase within 3 weeks later (Fig, 3).

\section{Immunocytochemistry detection}

\section{Ankle inflammation:}

A significant increase in the anteroposterior and transverse diameters of the left ankle of the MIAtreated rats and MIA- Chondrocytes treated rats at

Using phase imaging, induced adipocytes, osteocytes andall the measured time points post-injection (from chondrocytes showed a high local expression of mFABP4, day 7 to day 42) when compared with rats of hOsteocalcin and hAggrecan respectively in fluorescencecontrol group (Fig. 7A, 7B). However, there was image (Fig 4).

\section{Body Weight [BW] changes:}

Variations in body weight were checked weekly as evaluation parameters for the courses of stress and toxicity in the animals. The final body weight was significantly reduced in MIA-treated group compared with control group. Treatment of MIA group with Chondrocytes slightly increased body weight but still significantly reduced compared to control (Fig,5A). MIA- treatment significantly decreased body weight gain compared to control group while chondrocytes significantly increased body weight gain compared to MIA-treated group (Fig, 5B).

\section{Functional and behavioral evaluation:}

The total distance traveled in the arena during 5 minutes by MIA treated rats were significantly decreased at all time points compared to control rats. However, the total distance traveled by MIAChondrocytes treated rats were significantly increased in day 42 compared to MIA treated rats (Fig 6A). Rearing frequency of MIA treated rats was significantly lower in day $21,35,42$ and treatment with chondrocytes significantly increased it in day 21 and 42 (Fig 6B). no significant differences in either anteroposterior or transverse diameters of the left ankles were detected between MIA-treated rats and MIAChondrocytes treated rats at any time points after injection of MIA. Thus, Chondrocytes injection had no significant effects on ankle size.

\section{Microscopic evaluation:}

With hematoxylin and eosin, control group showed intact surface with smooth and glassy matrix, normal cartilage thickness and adequate chondrocytes density. However, group MIAtreated group showed surface discontinuity, erosion, vertical fissures and clefts and denudation. There was also a decrease in chondrocytes density. Furthermore, the articular cartilage layer was damaged, clear boundaries between cartilage layers were lost, and the arrangement of chondrocytes was more irregular compared to the control rats. On microscopic examination of articular cartilage of injected limbs of group C, there was an observable restoration of surface continuity, chondrocytes density, boundaries between layers and matrix basophilia. (Fig 8) 

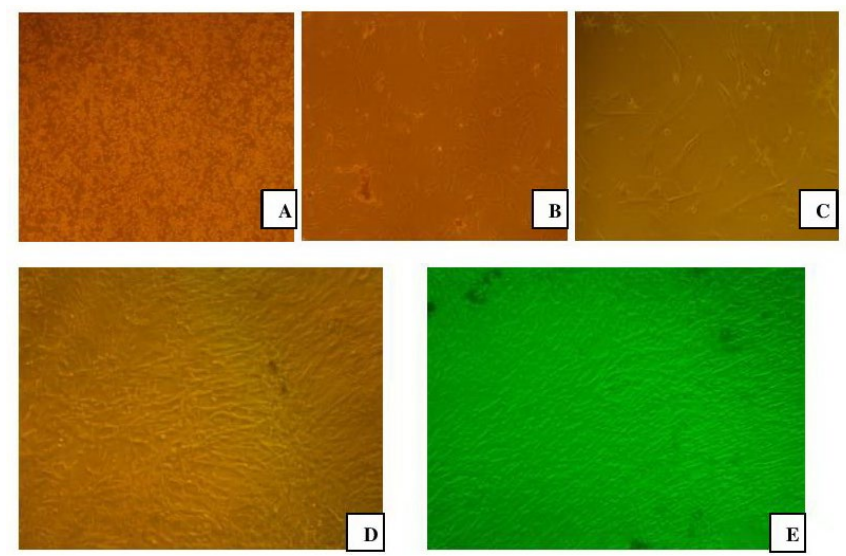

Fig 1: Inverted microscope image of Primary Culture of WJ-MSC (A): Day 7, discrete adherent cell layer with heterogeneous morphology (round and epithelioid cells) at the base of the flasks, and other cells floated in the media. (B): Day 10, adherent MSCs with floating non adherent cells. (C): Day 12 adherent fibroblast-like cells grew as spindle shaped cells at $50 \%-60 \%$ confluence. (D): Day 14 adherent fibroblast-like cells grew as spindle shaped cells at $60 \%-70 \%$ confluence. (E): phase contract microscope image at 14 day WJ-MSC.
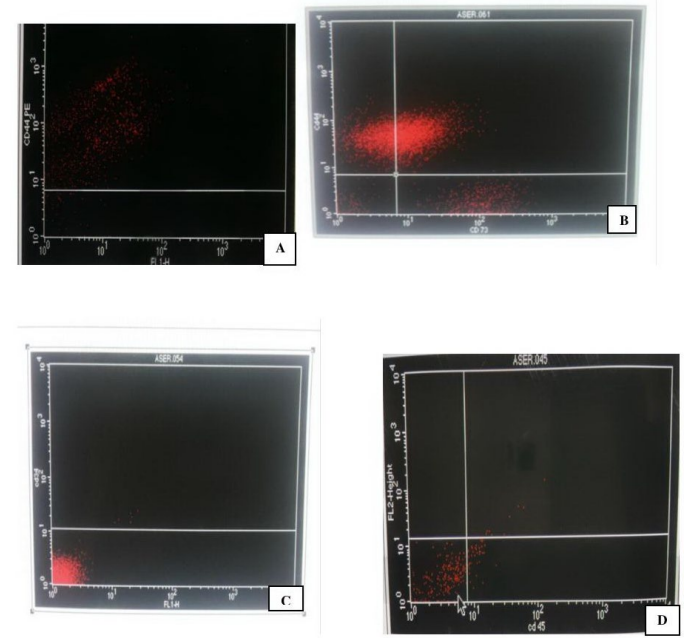

Fig 2: flowcytometric analysis of MSCs (A) MSCs were positive for CD44 PE (B) and double positive for CD44 PE and CD73 FITC (B) MSCs were negative for CD34 PE (C) negative for CD 45 FITC.

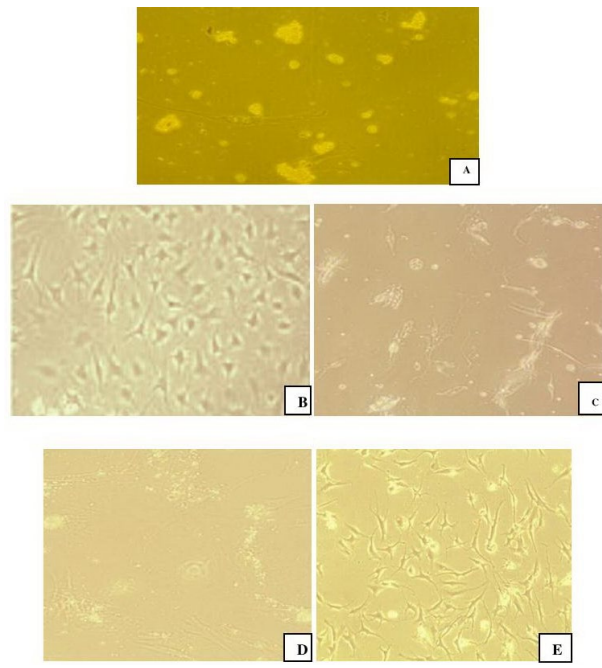

Fig 3: Trilling Differentiation of hWJ-MSCs (A): by inverted microscope [image x 200] Adipogenic differentiation with lipid accumulation (B) : by inverted microscope [image x 100] of differentiated osteocytes, (C) by inverted microscope [image $\mathrm{x} 200$ ] of differentiated osteocytes were acquired stellate- shape with cellular processes respectively (D): by inverted microscope [image $\mathrm{x}$ 100] Differentiated chondrocytes at day 7 (E): by inverted microscope [image x 100] chondrocytes 3 weeks post differentiation show globular shaped chondrocyte-like-cells. 

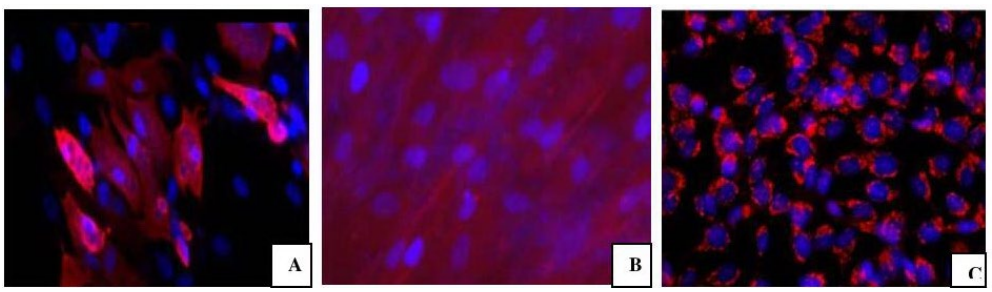

Fig 4: Microscopic image of immunocytochemistry staining of (A) adipocytes (B) osteocytes (C) chondrocytes. By fluorescence microscope [image x 200].
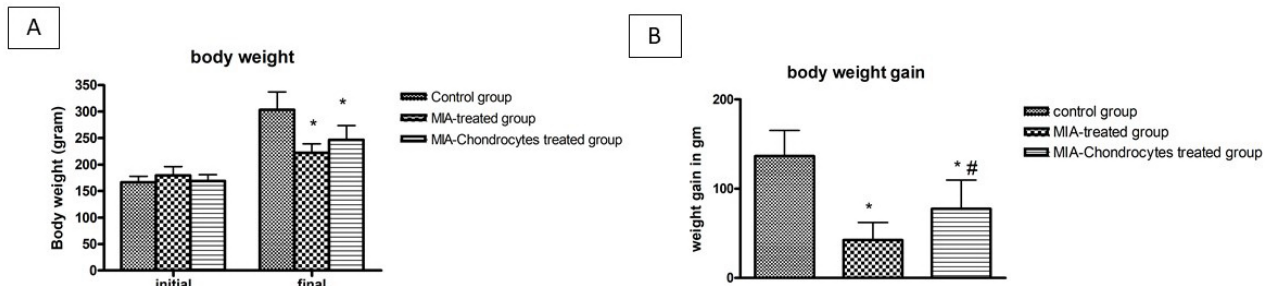

Fig 5: Body weight changes in different groups. (A) initial and final body weight of different groups. (B) body weight gain of different groups during the experiment. Data are presented as mean \pm SD; One- way ANOVA followed by Tukey's Multiple comparison test were used for further confirmation $(n=8)$. Significance was indicated as $* p<0.05$ vs. control group; \# $\mathrm{p}<0.05$ vs. MIA-treated group.
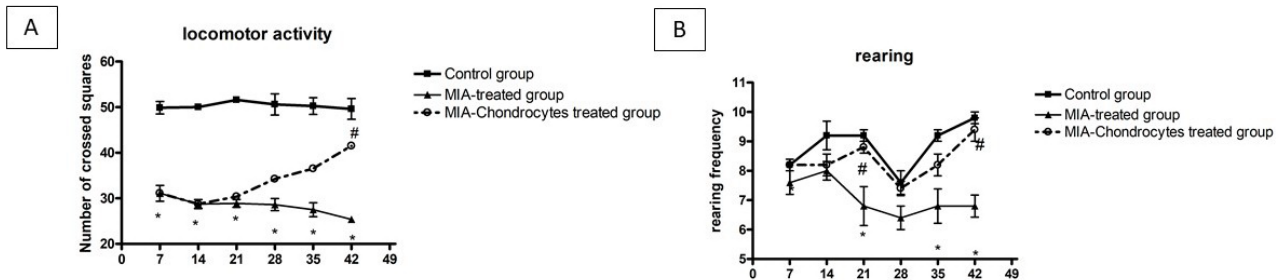

Fig 6: effect of chondrocytes on locomotor activity of MIA induced osteoarthritis in rats. $(n=8)$. Two way ANOVA and Bonferroni post hoc tests were used for analysis of locomotor activity. Significance was indicated as *p $<0.05$ vs. control group; \# $\mathrm{p}<0.05$ vs. MIA-treated group
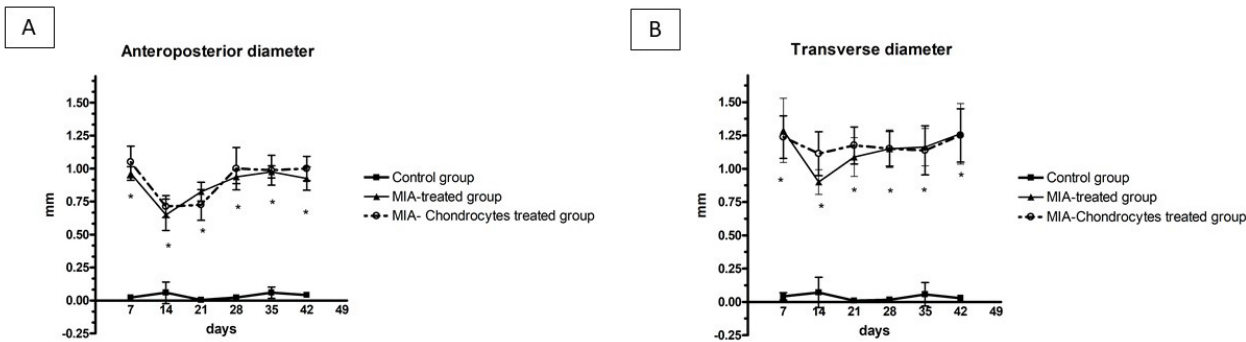

Fig 7: Effect of chondrocytes on ankle diameters of rats with MIA induced osteoarthritis. $(n=8)$. Two way ANOVA and Bonferroni post hoc tests were used for analysis of ankle diameters. (A) ankle anteroposterior diameters (B) ankle transverse diameter. Significance was indicated as $* \mathrm{p}<0.05$ vs. control group; $\# \mathrm{p}<0.05$ vs. MIA-treated group.
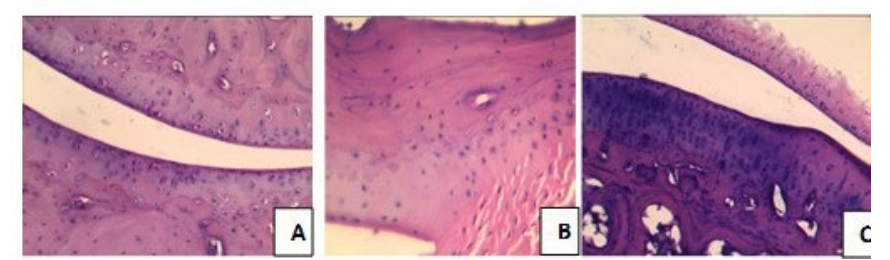

Fig:8 (A) light microscopic image (H\&E x200) of cartilage structure of a rat of group A. (B) light microscopic image (H\&E x200) showing osteoarthritic changes in a rat of group B.(C) light microscopic image (H\&E x200) of articular cartilage of a rat of group $\mathrm{C}$. 


\section{Histological scoring:}

The OARSI score was significantly lower (indicating less damage) in Group C with mean value $4.25 \pm 1.16$, than in Group B, mean value $13.38 \pm 3.11$.
By comparison between OARSI score of group B and group $\mathrm{C}$, there was highly significant difference $(\mathrm{P}<0.001)$ for the sake of group $\mathrm{B}$ (table $1)$.

Table1: Comparison between OARSI score of group B and group $C(n=8)$.

\begin{tabular}{||c|c|c|c|c|c||}
\hline Variable & & Group B & Group C & $\mathrm{T}$ & $\mathrm{P}$ \\
\hline \hline \multirow{3}{*}{ OARSI } & Min - Max & $9.0-18.0$ & $3.0-6.0$ & \multirow{3}{*}{7.59} & \multirow{2}{*}{$<0.001^{*}$} \\
\cline { 2 - 4 } & Mean \pm SD. & $13.38 \pm 3.11$ & $4.25 \pm 1.16$ & & \\
\cline { 2 - 4 } & Median & 13.50 & 4.0 & & \\
\hline
\end{tabular}

$\mathrm{t}$ and $\mathrm{p}$ values for paired samples T-test for comparing between OARSI score of group B and group C.

In the present study, monosodium

\section{Discussion}

Chondrocytes make up about only $5 \%$ of hyaline cartilage tissue. Chondrocytes are essential for cartilage function and homeostasis. These cells have mesenchymal origin, and are responsible for synthesizing cartilage extracellular matrix. Cartilage defect can arise as a concern of traumatic injury, leading to focal defects or through chronic degeneration [22].

Osteoarthritis is one of joint diseases which is most predominant and devastating type. It is associated with poor quality of life and high health care costs. OA is a complex disease affecting all joint tissues including cartilage, sub-chondral bone, synovium and fat pad, with accompanying inflammation that affects the entire joint leading to morbidity and disability in adults [23]. No approved therapeutic treatment protects articular cartilage, nor prevent the progression of $\mathrm{OA}$ However, Non-pharmacological, pharmacological, complementary alternative, and surgical intervention are the traditional therapeutic treatment for OA. These aimed to decrease pain, restore articular functions and quality of life [24]. iodoacetate (MIA) was used to induce monoarthritic rat model in the ankle joint. This model is valuable in studying ankle $\mathrm{OA}$ as cartilage degeneration is induced efficiently and the model has high reproducibility [25].

This present study revealed that intraarticular injection of MIA caused ankle swelling in the form of increase in the anteroposterior and transverse diameters of the left ankle at all postinjection time points which is in agreement with Jimbo et al. (2019) [25]. However, no improvement in ankle swelling was observed after injection of Chondrocytes. This may be explained by chronic MIA-induced inflammation.

In the present study, the body weight gain was lower in MIA-treated rats and increased after treatment with chondrocytes which could be explained by improvement of pain and locomotion which was reflected into ability to increase food intake. We examined our hypothesis by using the open field test which confirmed our explanation as MIA- induced OA decreased the locomotor activity of rats. However, rats treated with chondrocytes displayed significantly better 
locomotor recovery at the late stages of the treatment.

The more persistent impact of ankle inflammation on weight gain is, however, suggestive of increased nociception. Further studies will be required to test this hypothesis against alternative explanations.

Cellular therapy using stem cells are promised to revolutionize medicine as they having the ability to replace, repair or augment diseased tissues and organs [2]. Mesenchymal stem cells, the major stem cells for cell therapy, have been used due to their multilineage differentiation ability, their anti-inflammatory and immunoregulatory effect. This could be effective to rejuvenate and revitalize the damaged joints in OA, not only for cartilage repair but also for subchondral bone remodeling [15].WJ is easily accessible source of MSC. WJ-MSCs are maintained in an early embryologic stage with high proliferative capacity and primitive stemness properties [26]. In regenerative medicine WJMSCs is one of the most promising therapeutic agents for different pathologies, as WJ-MSCs, hold the ability to differentiate into a variety of lines and are slightly easier to harvest than other MSCs [26].

Umbilical cord Wharton's Jelly was used as a source of MSCs because of the easy noninvasive collection, no pain and no risk of infections with good sterilization precautions; this was agreed with [27]. Isolated MSCs were identified by: a) their ability to stick to to plastic flasks during cell culture; b) phenotypically defined set of surface markers including CD73, CD90, and CD105, and negative expression of CD14, CD19, CD34, CD45 and HLA-DR; c)
MSCs exhibited tri-lineage differentiation potentials and this done according to International Society for Cellular Therapy (ISCT) [12].

WJ-MSCs separated from the UC were expanded and the culture appeared homogeneous and cells presented their typical fusiform, fibroblast-like, morphology. These cells slowly reached 50\%-60\% confluence at about 14 days. This result agreed with [28] and [29] The flow cytometric analysis of WJ-MSCs showed that these cells were positive for human MSCs marker CD44 and CD73 which is unique for cells of the mesenchymal lineage, and negative for hematopoietic cell markers CD34 indicating that these cells were not of haematopoietic origin. This result agreed with Wang et al. (2004) [30] and Nagamura- Inoue and He. (2014) [29].

Confluent MSC cells were induced to differentiate into osteocytes, adipocytes and chondrocytes by culture in the presence of complete medium supplemented with osteogenic, adipogenic and chondrogenic supplements respectively. Mesenchymal stem cells induced with osteogenic supplement showed stellateshaped cells with cytoplasmic processes. This result agrees with Heino and Hentunen (2008) [31], according to Ding et al., (2011) [32] adipocytes differentiated from WJ-MSCs showed accumulation of lipid -rich vacuoles within cells. While WJ-MSCs induced with chondrogenic media showed round to polygonal shaped cells then exhibited elongated fibroblast like phenotypes and this agree with Schnabel et al., (2002) [33].

The differentiated cells were approved by immunocytochemistry analysis, adipocytes, osteocytes and chondrocytes showed positive immunoreactivity for anti-mFABP4, anti- 
hOsteocalcin and anti-hAggrecan, the nucleus stained blue and cytoplasm is counterstained red with DAPI and this agree with Aldridge et al., (2013) [34] Cheng et al., (2011) [35] and Tanthaisong et al., (2017) [10] respectively.

The articular cartilage of injected limbs showed restoration of surface continuity, chondrocytes density, boundaries between layers and matrix basophilia and The OARSI score was significantly lower. This effect due to not only the ability of differentiation but also self-replicate, secrete trophic molecules that promote cell-cell connection and diminish inflammation, apoptosis and fibrosis of damaged tissues, while stimulating tissue regeneration. These observations were in agreement with Al Faqeh et al., (2012) [36] who report that the animals endured injected chondrocytes, no sign of local inflammation or immobilization of the joint from the cell injection. Microscopic examination demonstrated reasonable cartilage surface. The superficial area was smooth without any sign of inflammation and almost similar cartilage thickness to the normal ankle. This changes to normal tissue occur due as the differentiated MSCs producing abundant extracellular matrix composed of cartilage specific molecules such as PGs, type II collagen, aggrecan and cartilage oligomeric matrix protein which are essential components of cartilage tissue [37] .

Because of the attractive benefits of MSCs including anti-inflammatory and immunomodulatory effects, the use of MSCs in regenerative medicine is encouraging, so that MSCs can treat a wide variety of illnesses. A substantial number of WJ-MSCs can be easily obtained after a few passages; the collection procedure is non-invasive and ethically acceptable and can be regarded for autologous and allogeneic use.

Limitations of this study include the short time of the study, few numbers of rats and lacking measurements of effects of MSCs on pain of OA. Therefore, we recommend performing more animal studies focusing on effect of MSC therapy on pain and compare with current therapy for OA to make MSC therapy a safe and effective therapeutic approach for osteoarthritis.

Conclusions: As WJ-MSCs exhibited tri-lineage differentiation potentials. Cell therapy with WJMSCs represents potentially new therapeutic strategy for treatment of osteoarthritis. This experimental trial revealed that intra-articular injections of chondrocytes induced MSCs stimulated cartilage rejuvenation and revitalization and reduced joint inflammation, leading to functional improvement of OA affecting joints.

\section{Acknowledgement:}

Authors wish to acknowledge the central lab in Faculty of Medicine, Menoufia University for using the facilities of tissue culture unit to perform this experiment.

\section{Disclosure Statement}

The authors have no conflicts of interest to declare.

\section{Funding Sources}

This study was self-funded from the authors and supported by Faculty of Medicine Menoufia University facilities.

\section{References}

1. Birchfield PC. Osteoarthritis overview. Geriatric Nursing 22 (3): 124- 131,2001.

2. Barry F and Murphy M. Mesenchymal stem cells in joint disease and repair. Nature Reviews Rheumatology 9:584- 94, 2013. 
3. Bhishek A and Doherty M. Diagnosis and clinical presentation of osteoarthritis. Rheumatic Disease of Clinics North America 39 (1): 45-66, 2013.

4. Zhang W, Ouyang H, Dass CR, Xu J.Current research on pharmacologic and regenerative therapies for osteoarthritis. Bone Research, 2016. $4 \quad .15040$ http://doi.org/ 10.1038/boneres.2015.40

5. Kong L, Zheng L Z, Qin L, Kevin K.W, Ho a.Role of mesenchymal stem cells in osteoarthritis treatment. Journal of Orthopaedic Translation 9: 89- 103, 2017.

6. Weissma I L. Stem cells are units of natural selection for tissue formation, for germline development, and in cancer development. Proceedings of the national academy of science U.S. A. 112: 8922-8928, 2015.

7. Sasaki M, Abe R, Fujita Y, Ando S, Inokuma D, Shimizu H. Mesenchymal stem cells are recruited into wounded skin and contribute to wound repair by transdifferentiation into multiple skin cell type. The Journal of Immunology 180: 2581-2587, 2008.

8. Lee DK and Song SU. Immunomodulatory mechanisms of mesenchymal stem cells and their therapeutic applications. Cellular Immunology 326: 68-76, 2018.

9. Lee OK, Kuo TK, Chen WM, Lee KD, Hsieh SL, Chen TH. Isolation of multipotent mesenchymal stem cells from umbilical cord blood. Blood 103:1669-1676, 2015.

10. Tanthaisong $\mathbf{P}$, Imsoonthornruksa $\mathbf{S}$, Ngernsoungnern $A$, Ngernsoungnern $P$ Ketudat-Cairns M , Parnpai R. Enhanced
Chondrogenic Differentiation of Human Umbilical Cord Wharton's Jelly Derived Mesenchymal Stem Cells by GSK-3 Inhibitors. PLoS ONE 12(1): e0168059, 2017.

11. McGuirk JP, Smith JR, Divine CL, Zuniga M, Weiss ML. Wharton's Jelly-Derived Mesenchymal Stromal Cells as a Promising Cellular Therapeutic Strategy for the Management of Graft-versus-Host Disease. Mineishi S, ed. Pharmaceuticals; 8(2):196220, 2015.

12. Dominici M, Le Blanc K, Mueller I, SlaperCortenbach I, Marini F, Krause D, Deans R, Keating A, Prockop Dj, Horwitz E. Minimal criteria for defining multipotent mesenchymal stromal cells. The International Society for Cellular Therapy position statement. Cytotherapy 8(4): 315-317, 2006.

13. Gärtner A, Pereira T, Gomes R, Luís AL, França ML, Geuna S, Armada-da-Silva P, Maurício AC. Mesenchymal Stem Cells from Extra-Embryonic Tissues for Tissue Engineering-Regeneration of the Peripheral Nerve. Advances in Biomaterials Science and Biomedical Applications: 466-498, 2013.

14. Mizuno H, Tobita M, Uysal AC. Concise review: adipose-derived stem cells as a novel tool for future regenerative medicine. Stem cells 30(5): 804-810, 2012.

15. Harrell C R, Markovicb BS, Fellabauma C, Arsenijevic A, Volarevic V. Mesenchymal stem cell-based therapy of osteoarthritis: Current knowledge and future perspectives Biomedicine \& Pharmacotherapy 109: 2318 2326, 2019.

16. Meirelles Lda S, Fontes AM, Covas DT, Caplan AI. Mechanisms involved in the 
therapeutic properties of mesenchymal stem cells. Cytokine \& Growth Factor Reviews 20 (5-6):419-427, 2009.

17. Petsa A, Gargani S, Felesakis A, Grigoriadis N, Grigoriadis I. Effectiveness of protocol for the isolation of Wharton's Jelly stem cells in large-scale applications. In Vitro Cellular \& Developmental Biology-Animal 45: 573- 586, 2009.

18. Colter DC, Sekiya I and Prockop DJ. Identification of a subpopulation of rapidly self-renewing and multipotential adult stem cells in colonies of human marrow stromal cells. Proceedings of the national academy of science 98: 7841-7845, 2001.

19. Guingamp C, Gegout-Pottie P, Philippe L, Terlain B, Patrick Netter P, Gillet P. MonoIodoacetate-Induced Experimental Osteoarthritis: a dose-response study of loss of mobility, morphology, and biochemistry. Arthritis \& Rheumatology 40 (9):1670-1679, 1997.

20. Braszko JJ, Wi'sniewski K., Kupryszewski G and Witczuk B. Psychotropic effects of angiotensin II and III in rats: Locomotor and exploratory vs. Cognitive behavior.

Behavioural Brain Research 25(3): 195-203, 1987.

21. Mapp PI, Sagar DR, Ashraf S, Burston JJ, Suri S, Chapman V, Walsh DA. Differences in structural and pain phenotypes in the sodium monoiodoacetate and meniscal transection models of osteoarthritis. Osteoarthritis and Cartilage 21(9):1336-1345, 2013.

22. Cottom JM and Maker JM. Cartilage allograft techniques and materials. Clinics in
Podiatric Medicine and Surgery 32(1): 93-98, 2015.

23. Lories RJ and Luyten FP. The bonecartilage unit in osteoarthritis. Nature Reviews Rheumatology 7: 43-49, 2011.

24. Evans CH. Advances in regenerative orthopedics, Mayo Clinic Proceedings 88 (11) 1323-1339, 2013.

25. Jimbo S, Terashima Y, Teramoto A, Takebayashi T Ogon I, Watanabe K, Sato T, Ichise N, Tohse N, Yamashita, T. Antinociceptive effects of hyaluronic acid on monoiodoacetate-induced ankle osteoarthritis in rats. Journal of Pain Research 12: 191-200, 2019.

26. Kim DW, Staples M, Shinozuka K, Pantcheva P, Kang SD, Borlongan C V. Wharton's Jelly-Derived Mesenchymal Stem Cells: Phenotypic Characterization and Optimizing Their Therapeutic Potential for Clinical Applications. International Journal of Molecular Sciences 14(6): 11692-11712, 2013.

27. Riordan NH, MoralesI , Fernández G, Allen N, Fearnot NE, Leckrone ME, Markovich DJ, Mansfeld D , Avila D, Patel AN, Kesari S and Rodriguez JP. Clinical feasibility of umbilical cord tissue-derived mesenchymal stem cells in the treatment of multiple sclerosis. Journal of translational medicine 16(1): 57, 2018.doi:10.1186/s12967-0181433-7

28. Koliakos I, Tsagias N, Karagiannis V. Mesenchymal cells isolation from Wharton's jelly, in perspective to clinical applications. Journal of Biological Research-Thessaloniki 16: 194 - 201, 2011. 
29. Nagamura-Inoue $\mathbf{T}$ and $\mathrm{He} \boldsymbol{H}$. Umbilical cord-derived mesenchymal stem cells: Their advantages and potential clinical utility. World Journal of Stem Cells 26 6(2): 195-202, 2014.

30. Wang HS, Hung SC, Peng ST, Huang CC, Wei HM, Guo YJ, Fu YS, Lai MC, Chen CC. Mesenchymal stem cells in the Wharton's jelly of the human umbilical cord. Stem cells 22 (7): 1330-1337, 2004.

31. Heino TJ and Hentunen TA. Differentiation of osteoblasts and osteocytes from mesenchymal stem cells. Current Stem Cell Research \& Therapy 3(2):131-45, 2008.

32. Ding DC, Shyu WC and Lin SZ. Mesenchymal Stem Cells. Cell Transplantation 20: 5-14, 2011.

33. Schnabel M, Marlovits S, Eckhoff G, Fichtel I, Gotzen L, Vecsei V, Schlegel J. Dedifferentiation-associated changes in morphology and gene expression in primary human articular chondrocytes in cell culture. Osteoarthritis and Cartilage 10: 6270, 2002.

34. Aldridge A, Kouroupis D, Churchman S, English A, Ingham E and Jones E. Assay validation for the assessment of adipogenesis of multipotential stromal cells - $\mathrm{a}$ direct comparison of four different methods. Cytotherapy 15: 89-101, 2013.

35. Cheng H, Qiu L, Ma J, Zhang H, Cheng M, Li W, Zhao X, Liu K. Replicative senescence of human bone marrow and umbilical cord derived mesenchymal stem cells and their differentiation to adipocytes and osteoblasts. Molecular Biology Reports 38(8):5161-5168, 2011.

36. Al Faqeh H, Nor Hamdan BM, Chena HC, Aminuddin BS, Ruszymah BH. The potential of intra-articular injection of chondrogenicinduced bone marrow stem cells to retard the progression of osteoarthritis in a sheep model . Experimental Gerontology 47 (6): 458-464, 2012.

37. Nam Y, Rim YA, Jung SM, Ju JH. Cord blood cell-derived iPSCs as a new candidate for chondrogenic differentiation and cartilage regeneration. Stem Cell Research \& Therapy 8(1):16., 2017. DOI 10.1186/s13287-0170477-6 